\title{
Blood lead, cadmium and mercury in relation to homocysteine and C-reactive protein in women of reproductive age: a panel study
}

Anna Z. Pollack ${ }^{1 *}$, Sunni L. Mumford², Lindsey Sjaarda², Neil J. Perkins², Farah Malik², Jean Wactawski-Wende ${ }^{3}$ and Enrique F. Schisterman ${ }^{2}$

\begin{abstract}
Background: To examine the relationship between cadmium, lead, and mercury concentrations with high-sensitivity C-reactive protein (hs-CRP) and homocysteine in women.

Methods: Metals were measured at enrollment in whole blood. Homocysteine and hs-CRP were measured in one $(N=9)$ or two $(N=250)$ menstrual cycles up to 3 and 8 times per cycle, respectively. Linear mixed models with inverse probability of exposure weights to account for time varying confounding were used and models were stratified by dietary and serum vitamin status (dietary: vitamin $B_{6}, B_{12}$, folate; serum: folate).

Results: Geometric mean (95\% confidence interval (CI)) concentrations for cadmium, lead, and mercury were $0.29(0.26-0.31) \mu \mathrm{g} / \mathrm{L}, 0.91(0.86-0.96) \mu \mathrm{g} / \mathrm{dL}$, and $1.05(0.93-1.18) \mu \mathrm{g} / \mathrm{L}$, respectively. Lead was associated with increased homocysteine $(0.08 ; 95 \% \mathrm{Cl}: 0.01,0.15)$ and this persisted among those in the lower three quartiles of consumption of vitamin $B_{6}, B_{12}$, folate, and serum folate but was not significant among those in the upper quartile. No associations were observed between metals and hs-CRP.
\end{abstract}

Conclusions: Blood lead was associated with increased homocysteine in a cohort of healthy, premenopausal women but these associations did not persist among those consuming $\geq 75$ th percentile of essential micronutrients. Cadmium, lead, and mercury were not associated with hs-CRP concentrations.

Keywords: C-reactive protein, Cadmium, Homocysteine, Inflammation, Lead, Mercury, Women

\section{Background}

A growing body of research points to associations between metals and the inflammation markers homocysteine and high sensitivity C-reactive protein (hsCRP). Lead may increase homocysteine concentrations by interacting with thiols involved in one-carbon metabolism of homocysteine production [1]. Limited evidence suggests an association between cadmium, lead, and homocysteine in US adults [2], and between mercury and homocysteine in children [3]. Among

\footnotetext{
* Correspondence: apollac2@gmu.edu

${ }^{1}$ Department of Global and Community Health, College of Health and Human Services, George Mason University, 4400 University Drive MS5B7, Fairfax, VA 22030, USA

Full list of author information is available at the end of the article
}

older adults, populations with higher lead concentrations, and occupationally exposed workers, lead was associated with increased homocysteine concentrations $[1,4-6]$. Cadmium was linked with hs-CRP in a study of cardiovascular events [7], in a cross-sectional study of US adults [8], and this association was attenuated among those with higher dietary antioxidant intake [9]. The blood lead-homocysteine association was stronger in older men with below-median micronutrient concentrations [10] and in a cross-sectional study of US adults [5]. Micronutrients play an important role in homocysteine/methionine metabolism, and $\mathrm{B}$ vitamins in particular can decrease homocysteine concentrations [11]. Although little is known about the modifying role that dietary micronutrient 
intake may have, these findings suggest that micronutrient levels may play an important role in the relationship between metals and homocysteine and hs-CRP.

However, research on homocysteine and hs-CRP in relation to metals concentrations in reproductive-aged women is limited. Cardiovascular disease (CVD) is the leading cause of death in U.S. women [12, 13] and modifiable factors offer important avenues for prevention. Total plasma homocysteine (tHcy) and hs-CRP are considered CVD risk biomarkers [14-16]. Both hs-CRP and homocysteine reflect distinct cellular processes that may contribute to inflammation and thus evaluating both biomarkers may provide greater insight as the specific mechanism by which metals influence CVD risk remains unknown.

Given limited research in reproductive aged women, our objective was to examine associations between cadmium, lead, and mercury with homocysteine and hs-CRP and to examine these associations stratified by methyl-donor nutrient dietary consumption (folate, vitamin $B_{6}$ and $B_{12}$ ) and serum folate concentrations in healthy, reproductive-aged women. Testing of this hypothesis was made possible by utilizing an existing high-quality data set.

\section{Methods}

The BioCycle Study was a prospective cohort study that followed 259 healthy, premenopausal women aged 18-44 from Buffalo, New York, for up to two menstrual cycles. The majority of women participated in consecutive menstrual cycles. Of the 24 nonconsecutive cycles, the median time interval between cycles was 30 days (IQR 27, 45), and the mean interval was 43.5 days. Recruitment occurred from 2005 to 2007. Details regarding the BioCycle Study have been published [17]. Briefly, participants provided selfreported demographics and information including reproductive history, smoking, and alcohol consumption. Participants were selected to be free of chronic disease and were not taking medications. Height and weight were measured using standard protocols by trained study staff to determine body mass index (BMI) $\left(\mathrm{kg} / \mathrm{m}^{2}\right)$. The University at Buffalo Health Sciences Institutional Review Board (IRB) approved the study and served as the IRB designated by the $\mathrm{NIH}$ for this study under a reliance agreement. All participants provided written informed consent.

\section{High sensitivity C-reactive protein and homocysteine measurement}

Fasting blood was drawn in the morning during clinic visits, which were scheduled to correspond to approximately the second day of menstruation, mid- and latefollicular phase, 2 days around expected ovulation, and early, mid-, and late-luteal phase in each cycle. Within 90 min of the blood draw, all samples were processed and frozen at $-80{ }^{\circ} \mathrm{C}$ and were shipped as complete individual cycle batches to the analytical laboratory. Ninety-four percent of study participants attended at least 7 of 8 scheduled clinic visits per cycle and 100\% completed at least 5 clinic visits per cycle.

C-reactive protein was measured at each clinic visit in serum using high sensitivity methods on the IMMULITE 2000 (Siemens Medical Solutions Diagnostics, Deerfield, IL, USA), a chemiluminescent immunoassay sensitive to $0.3 \mathrm{mg} / \mathrm{l}$ [18]. Hs-CRP is considered a more sensitive assay for cardiovascular disease prediction compared with CRP according to Centers for Disease Control and Prevention and the American Heart Association guidelines $[19,20]$. Homocysteine was measured 3 times each cycle (mid-follicular, ovulation, and mid-luteal menstrual cycle phase) in serum at the Kaleida Laboratory in Buffalo, New York, by chemiluminescence with Siemens IMMULITE 2000 homocysteine competitive immunoassay (coefficient of variation $[\mathrm{CV}]<10.4 \%$ ).

\section{Metals measurement}

Whole blood was collected at study enrollment, which occurred approximately 2 weeks prior to the first cycle study visit. Samples were collected in purple-top tubes that were pre-screened to be free of trace metal contamination (Becton, Dickinson, and Company, Franklin Lakes, NJ). Centers for Disease Control (CDC) provided the collection tubes and protocols for collection, storage, and transport. Samples were refrigerated and sent to the Division of Laboratory Sciences, National Center for Environmental Health, for lead, cadmium, and mercury assessment by inductively coupled plasma mass spectrometry (ICP-MS). The interassay CV for cadmium, lead, and mercury were $4.3,2.6$ and $3.2 \%$, respectively. Machine read values were used and values below the limit of detection (LOD) were not substituted to minimize bias [21, 22]. The LODs for cadmium, lead, and mercury were $0.20 \mu \mathrm{g} / \mathrm{L}$ $(25 \%<\mathrm{LOD}), 0.25 \mu \mathrm{g} / \mathrm{dL}(0 \%<\mathrm{LOD})$, and $0.30 \mu \mathrm{g} / \mathrm{L}$ $(12 \%<$ LOD), respectively.

\section{Nutrient and hormone assessment}

Nutrient intake of dietary folate, vitamin $B_{6}, B_{12}$, fish consumption, and omega- 3 fatty acids, were assessed four times per cycle using a 24-h dietary recall questionnaire, for up to eight recalls total. Mean fish consumption was determined for each participant. As these components were measured by the same instrument, recall or other errors may be interdependent. Dietary intake data were collected and analyzed using the Nutrition Data System for Research software version 2005 developed by the Nutrition Coordinating Center, University of Minnesota. Eighty-seven percent of participants completed four 
dietary recalls per cycle and 99\% completed 3 per cycle. Serum folate was measured at each clinic visit by the University of Minnesota from stored blood samples using a competitive protein binding assay and was performed with an Roche Elecsys 2010 Analyzer using the Roche Folate Gen 3 reagent (Roche Diagnostics, Indianapolis, Indiana) to detect 5-methyl tetrahydrofolate (THF), 5formyl THF, and folic acid within the serum samples for each woman ( $\mathrm{CV}<9.5 \%)$. Reproductive hormones were measured in fasting serum samples from all visits (5-8 visits/cycle) at the Kaleida Health Center for Laboratory Medicine (Buffalo, NY). Estradiol, progesterone, FSH, and LH were measured by solid-phase competitive chemiluminescent enzymatic immunoassay on the DPC Immulite 2000 analyzer (Siemens Medical Solutions Diagnostics) by Specialty Laboratories and CVs were $<10 \%$ for estradiol, $<5 \%$ for $\mathrm{LH}$ and $\mathrm{FSH}$, and $<14 \%$ for progesterone.

\section{Statistical methods}

Descriptive statistics were evaluated for all covariates by homocysteine and hs-CRP dichotomized at their medians (hs-CRP: $0.8 \mathrm{mg} / \mathrm{L}$; homocysteine: $5.9 \mu \mathrm{mol} / \mathrm{L}$ ). Categorical variables were assessed using Pearson ChiSquare or Fisher exact tests while continuous variables were assessed using t-tests. Linear mixed models with log-transformed hs-CRP, homocysteine, and metals were run separately for each metal. Values greater than $10 \mathrm{mg} / \mathrm{L}$ of hs-CRP were excluded, as those values are indicative of current infection. A first-order autoregressive moving-average structure was specified for the correlation matrix. Random intercepts accounted for variation in hs-CRP and homocysteine concentrations between women and all measured values for hs-CRP and homocysteine were used. The mixed models accounted for the day of the menstrual cycle and cycle number for each woman. Inverse probability of exposure weighted models were implemented to account for time-varying confounding by micronutrient (Vitamin $\mathrm{B}_{6}, \mathrm{~B}_{12}$, dietary and serum folate) and reproductive hormone concentrations (estradiol, progesterone, luteinizing hormone (LH), and follicle stimulating hormone (FSH)) [23, 24]. Confounders were selected based on a review of the literature and associations observed in descriptive analyses. Age, race (black, white, Asian, other), BMI, parity, smoking, and day of menstrual cycle were included in adjusted models. Results from continuous models are presented as a percent change in the outcome values per one-percent increase in the non-transformed exposure. To evaluate the potential for an interaction between metal exposure with homocysteine and hs-CRP by intake of $\mathrm{B}$ vitamins and folate or serum folate, models were stratified at the 75 th percentile.

To examine the robustness of our findings, sensitivity analyses were run. First, a co-exposure model with all metals included together in the same model and a composite metal exposure category was created whereby if a participant was in the high category of exposure for any two metals, she was considered as high, whereas if a participant was in the low category for any two metals, she was considered low and otherwise was in the middle category of exposure. Tertiles were categorized as, cadmium: $0.04-0.22 \mu \mathrm{g} / \mathrm{L}, 0.23-0.36 \mu \mathrm{g} / \mathrm{L}$, and $0.37-3.1 \mu \mathrm{g} / \mathrm{L}$; lead: $0.31-0.72 \mu \mathrm{g} / \mathrm{dL}, 0.73-1.0 \mu \mathrm{g} / \mathrm{dL}$, 1.1-6.2 $\mu \mathrm{g} / \mathrm{dL}$; mercury: 0-0.8 $\mu \mathrm{g} / \mathrm{L}, 0.83-1.6 \mu \mathrm{g} / \mathrm{L}, 1.7-$ $9.9 \mu \mathrm{g} / \mathrm{L}$. A secondary analysis with fish consumption and omega-3 fatty acids were included as potential confounders both together and separately in the mercury exposure models. Statistical significance was defined as $p<0.05$ and SAS version 9.4 was used for all analysis (SAS Institute, Cary, NC, USA).

\section{Results}

Overall, women were young (mean 27.4, SD 8.2 years), of normal BMI $\left(24.1\right.$, SD $\left.3.9 \mathrm{~kg} / \mathrm{m}^{2}\right)$, did not smoke (96\%), and were nulliparous (74\%) (Table 1). Geometric mean concentrations of cadmium, lead, and mercury were $0.29 \mu \mathrm{g} / \mathrm{L}, 0.93 \mu \mathrm{g} / \mathrm{dL}$, and $1.03 \mu \mathrm{g} / \mathrm{L}$, respectively. Median concentrations of hs-CRP and homocysteine were $0.56 \mathrm{mg} / \mathrm{L}$ (range $0.10,151.0$ ) and $5.9 \mu \mathrm{mol} / \mathrm{L}$ (range $2.0,12.9$ ). No participants had levels that classified them as having high homocysteine $(>15 \mu \mathrm{mol} / \mathrm{L})$. One percent of hs-CRP values were $>10 \mathrm{mg} / \mathrm{L}$, indicative of current infection and $9 \%$ of values were $>3 \mathrm{mg} / \mathrm{L}$, the American Heart Association cutoff for elevated risk [25]. The majority of participants identified as white (57\%). Those with hs-CRP concentrations above the median were older (29.5 vs. 25.4 years), had higher BMI (25.9 vs. $22.5 \mathrm{~kg} / \mathrm{m}^{2}$ ), and were more often parous (36 vs. 17\%) compared to women with lower hs-CRP concentrations. Those with homocysteine concentrations above the median had higher concentrations of lead, and lower serum folate, dietary folate, vitamin $\mathrm{B}_{12}$ (Table 1).

Each percent increase in lead concentrations was associated with a $0.08 \mu \mathrm{mol} / \mathrm{L}(95 \% \mathrm{CI}: 0.01,0.15)$ difference in homocysteine concentrations after adjustment for relevant confounding factors and inverse probability of exposure weights to account for timevarying confounding factors (Table 2). In unadjusted models, cadmium was associated with increased homocysteine $0.04 \mu \mathrm{mol} / \mathrm{L}$ (95\% CI: 0.001, 0.09) but this association was not robust to adjustment for relevant confounding factors. Lead, cadmium, and mercury were not associated with hs-CRP.

Potential modification of the associations between blood metals concentrations with hs-CRP and homocysteine by levels of dietary vitamins $B_{6}, B_{12}$, and folate, and serum folate were examined by dichotomizing vitamin $\mathrm{B}$ and folate at the 75 th 
Table 1 Participant characteristics by high-sensitivity C-reactive protein (hs-CRP) and plasma homocysteine (tHcy) in women, Buffalo, NY

\begin{tabular}{|c|c|c|c|c|c|c|c|}
\hline & $\begin{array}{l}\text { Population } \\
(n=259)\end{array}$ & $\begin{array}{l}\text { hs-CRP } \\
<0.56 \mathrm{mg} / \mathrm{L}^{\dagger}\end{array}$ & $\begin{array}{l}\text { hs-CRP } \\
\geq 0.56 \mathrm{mg} / \mathrm{L}^{+}\end{array}$ & $p$-value ${ }^{\ddagger}$ & $\begin{array}{l}\text { tHcy } \\
<5.9 \mu \mathrm{mol} / \mathrm{L}^{+}\end{array}$ & $\begin{array}{l}\text { tHcy } \\
\geq 5.9 \mu \mathrm{mol} / \mathrm{L}^{\dagger}\end{array}$ & $p$-value \\
\hline Age, years & $27.4 \pm 8.2$ & $25.4 \pm 7.3$ & $29.5 \pm 8.6$ & $<0.01$ & $28.1 \pm 8.4$ & $26.9 \pm 8.1$ & 0.28 \\
\hline $\mathrm{BMI}, \mathrm{kg} / \mathrm{m}^{2}$ & $24.1 \pm 3.9$ & $22.5 \pm 2.8$ & $25.9 \pm 4.1$ & $<0.01$ & $23.9 \pm 3.7$ & $24.3 \pm 4.0$ & 0.36 \\
\hline Cadmium, $\mu \mathrm{g} / \mathrm{L}^{\mathrm{a}}$ & $\begin{array}{l}0.29 \\
(0.26,0.31)\end{array}$ & $\begin{array}{l}0.29 \\
(0.26,0.32)\end{array}$ & $\begin{array}{l}0.28 \\
(0.25,0.32)\end{array}$ & 0.85 & $\begin{array}{l}0.26 \\
(0.23,0.30)\end{array}$ & $\begin{array}{l}0.30 \\
(0.27,0.34)\end{array}$ & 0.12 \\
\hline Lead, $\mu \mathrm{g} / \mathrm{dL}^{\mathrm{a}}$ & $\begin{array}{l}0.91 \\
(0.86,0.96)\end{array}$ & $\begin{array}{l}0.92 \\
(0.85,1.00)\end{array}$ & $\begin{array}{l}0.90 \\
(0.84,0.98)\end{array}$ & 0.78 & $\begin{array}{l}0.83 \\
(0.77,0.90)\end{array}$ & $\begin{array}{l}0.97 \\
(0.90,1.05)\end{array}$ & $<0.01$ \\
\hline Mercury, $\mu g / L^{a}$ & $\begin{array}{l}1.05 \\
(0.93,1.18)\end{array}$ & $\begin{array}{l}1.03 \\
(0.86,1.22)\end{array}$ & $\begin{array}{l}1.07 \\
(0.90,1.28)\end{array}$ & 0.72 & $\begin{array}{l}1.14 \\
(0.94,1.37)\end{array}$ & $\begin{array}{l}0.99 \\
(0.84,1.17)\end{array}$ & 0.28 \\
\hline Estradiol, pg/mL ${ }^{\mathrm{a}} \mathrm{b}$ & $\begin{array}{l}51.22 \\
(48.60,53.98)\end{array}$ & $\begin{array}{l}50.2 \\
(45.4,55.5)\end{array}$ & $\begin{array}{l}50.4 \\
(44.4,56.7)\end{array}$ & 0.95 & $\begin{array}{l}52.6 \\
(46.4,59.6)\end{array}$ & $\begin{array}{l}48.9 \\
(44.3,53.9)\end{array}$ & 0.35 \\
\hline $\mathrm{FSH}, \mathrm{mlU} / \mathrm{mL}^{\mathrm{a}, \mathrm{b}}$ & $\begin{array}{l}6.59 \\
(6.39,6.79)\end{array}$ & $\begin{array}{l}6.51 \\
(6.16,6.88)\end{array}$ & $\begin{array}{l}6.50 \\
(6.03,7.00)\end{array}$ & 0.97 & $\begin{array}{l}6.40 \\
(5.92,6.90)\end{array}$ & $\begin{array}{l}6.58 \\
(6.21,6.96)\end{array}$ & 0.94 \\
\hline $\mathrm{LH}, \mathrm{ng} / \mathrm{mL}^{\mathrm{a}, \mathrm{c}}$ & $\begin{array}{l}9.55 \\
(8.87,10.30)\end{array}$ & $\begin{array}{l}9.35 \\
(8.14,10.74)\end{array}$ & $\begin{array}{l}9.54 \\
(8.14,11.18)\end{array}$ & 0.85 & $\begin{array}{l}9.39 \\
(8.11,10.88)\end{array}$ & $\begin{array}{l}9.48 \\
(8.18,10.99)\end{array}$ & 0.93 \\
\hline Progesterone, $\mathrm{ng} / \mathrm{ml}^{\mathrm{a}, \mathrm{d}}$ & $\begin{array}{l}6.16 \\
(5.60,6.77)\end{array}$ & $\begin{array}{l}5.18 \\
(4.18,6.43)\end{array}$ & $\begin{array}{l}6.41 \\
(5.33,7.71)\end{array}$ & 0.14 & $\begin{array}{l}6.27 \\
(5.20,7.56)\end{array}$ & $\begin{array}{l}5.22 \\
(4.20,6.49)\end{array}$ & 0.20 \\
\hline Serum folate, $\mathrm{ng} / \mathrm{ml}^{\mathrm{a}}$ & $\begin{array}{l}19.89 \\
(19.40,20.38)\end{array}$ & $\begin{array}{l}19.8 \\
(18.9,20.8)\end{array}$ & $\begin{array}{l}20.0 \\
(19.1,21.0)\end{array}$ & 0.70 & $\begin{array}{l}21.3 \\
(20.2,22.4)\end{array}$ & $\begin{array}{l}19.1 \\
(18.3,19.9)\end{array}$ & $<0.01$ \\
\hline Dietary folate, $\mu \mathrm{g} /$ day $^{\mathrm{a}}$ & $318.55(303.0,334.9)$ & $\begin{array}{l}331.4 \\
(300.5,365.5)\end{array}$ & $\begin{array}{l}311.7 \\
(281.0,345.8)\end{array}$ & 0.40 & $\begin{array}{l}349.2 \\
(311.8,391.2)\end{array}$ & $\begin{array}{l}305.4 \\
(278.9,334.4)\end{array}$ & $<0.01$ \\
\hline Dietary Vitamin $B_{6}, \mathrm{mg} /$ day $^{a}$ & $\begin{array}{l}1.25 \\
(1.19,1.32)\end{array}$ & $\begin{array}{l}1.32 \\
(1.19,1.45)\end{array}$ & $\begin{array}{l}1.26 \\
(1.13,1.42)\end{array}$ & 0.60 & $\begin{array}{l}1.38 \\
(1.23,1.54)\end{array}$ & $\begin{array}{l}1.24 \\
(1.12,1.37)\end{array}$ & 0.17 \\
\hline Dietary Vitamin $B_{12}, \mu \mathrm{g} /$ day $^{\mathrm{a}}$ & $\begin{array}{l}2.53 \\
(2.33,2.76)\end{array}$ & $\begin{array}{l}2.67 \\
(2.24,3.18)\end{array}$ & $\begin{array}{l}2.64 \\
(2.24,3.10)\end{array}$ & 0.92 & $\begin{array}{l}3.06 \\
(2.16,3.58)\end{array}$ & $\begin{array}{l}2.42 \\
(2.05,2.86)\end{array}$ & 0.04 \\
\hline Alcohol, litre/day ${ }^{a}$ & $\begin{array}{l}0.14 \\
(010,0.21)\end{array}$ & $\begin{array}{l}0.13 \\
(0.06,0.27)\end{array}$ & $\begin{array}{l}0.20 \\
(0.09,0.47)\end{array}$ & 0.43 & $\begin{array}{l}0.14 \\
(0.05,0.38)\end{array}$ & $\begin{array}{l}0.17 \\
(0.09,0.33)\end{array}$ & 0.76 \\
\hline Total calories, Kcal/day ${ }^{a}$ & $\begin{array}{l}1482.8 \\
(1433,1534)\end{array}$ & $\begin{array}{l}1454 \\
(1355,1560)\end{array}$ & $\begin{array}{l}1487 \\
(1378,1605)\end{array}$ & 0.67 & $\begin{array}{l}1562 \\
(1432,1702)\end{array}$ & $\begin{array}{l}1415 \\
(1328,1508)\end{array}$ & 0.06 \\
\hline \multicolumn{8}{|l|}{ Race $^{e}$} \\
\hline White & $148(57)$ & $71(55)$ & $77(64)$ & 0.18 & $56(56)$ & $92(61)$ & 0.24 \\
\hline Black & $51(20)$ & $26(20)$ & $25(21)$ & & $26(26)$ & $25(17)$ & \\
\hline Asian & $35(14)$ & $24(19)$ & $11(9)$ & & $11(11)$ & $24(16)$ & \\
\hline Other & $25(10)$ & $8(6)$ & $7(6)$ & & $7(7)$ & $8(5)$ & \\
\hline \multicolumn{8}{|l|}{ Smoking ${ }^{e}$} \\
\hline No/former & 249 (96) & 125 (97) & $114(95)$ & 0.53 & $98(98)$ & $141(54)$ & 0.30 \\
\hline Current & $10(4)$ & $4(3)$ & $6(5)$ & & $2(2)$ & $8(5)$ & \\
\hline \multicolumn{8}{|l|}{ Parity ${ }^{e}$} \\
\hline 0 & $187(74)$ & $109(83)$ & $78(64)$ & $<0.01$ & $66(65)$ & $121(80)$ & $<0.01$ \\
\hline$\geq 1$ & $66(26)$ & $22(17)$ & $44(36)$ & & $36(35)$ & $30(20)$ & \\
\hline
\end{tabular}

${ }^{a}$ Geometric mean (95\% confidence limits) ${ }^{b}$ Day $7^{c}$ Day $14^{d}$ Day 22

${ }^{e}$ Categorical variables [race, smoking, parity] expressed as $\mathrm{n}(\%)$

${ }^{\dagger}$ thcy and hs-CRP concentrations dichotomized on cycle 1 day 7

${ }^{\ddagger}$ Continuous variables compared using t-test; categorical variables compared using Chi-square test or Fisher's exact test

percentile and examining stratified associations. Lead was consistently associated with an increase in homocysteine concentrations among those with micronutrient levels below the 75th percentile (Table 2). Notably, among those with micronutrient levels $\geq 75$ th percentile, lead was not significantly associated with homocysteine (Table 2). Mercury was associated with decreased concentrations of homocysteine among those with serum folate $<24.49 \mathrm{ng} / \mathrm{ml},-0.03 \mu \mathrm{mol} / \mathrm{L}$ (95\% CI: -0.06, -0.005) (Table 2). Micronutrient levels did not modify the association between metals and hs-CRP. 
Table 2 Cadmium, lead, and mercury association with homocysteine and c-reactive protein overall and stratified by dietary and serum micronutrients at the 75 th percentile

\begin{tabular}{|c|c|c|c|c|}
\hline & \multicolumn{2}{|l|}{$\begin{array}{l}\text { hs-CRP }(\mathrm{mg} / \mathrm{L})^{\mathrm{a}} \\
\beta(95 \% \mathrm{Cl})\end{array}$} & \multicolumn{2}{|l|}{$\begin{array}{l}\text { tHcy }(\mu \mathrm{mol} / L)^{\mathrm{a}} \\
\beta(95 \% \mathrm{Cl})\end{array}$} \\
\hline & Unadjusted & Adjusted $^{\mathrm{b}}$ & Unadjusted & Adjusted $^{\mathrm{b}}$ \\
\hline Cadmium $^{\mathrm{a}}, \mu \mathrm{g} / \mathrm{I}$ & $-0.03(-0.22,0.15)$ & $-0.08(-0.25,0.08)$ & $0.04(0.001,0.09)$ & $0.03(-0.01,0.08)$ \\
\hline Lead $^{a}, \mu \mathrm{g} / \mathrm{dl}$ & $-0.05(-0.31,0.21)$ & $-0.05(-0.28,0.19)$ & $0.10(0.04,0.16)$ & $0.08(0.01,0.15)$ \\
\hline \multirow[t]{3}{*}{ Mercury $^{a}, \mu \mathrm{g} / \mathrm{I}$} & $-0.02(-0.14,0.11)$ & $0.02(-0.09,0.12)$ & $-0.01(-0.04,0.01)$ & $-0.03(-0.05,0.003)$ \\
\hline & \multicolumn{4}{|l|}{ Dietary Vitamin $\mathrm{B}_{6}$} \\
\hline & $<1.84 \mathrm{mg} / \mathrm{day}^{\mathrm{b}}$ & $\geq 1.84 \mathrm{mg} / \mathrm{day}^{\mathrm{b}}$ & $<1.84 \mathrm{mg} / \mathrm{day}^{\mathrm{b}}$ & $\geq 1.84 \mathrm{mg} / \mathrm{day}^{\mathrm{b}}$ \\
\hline Cadmium $^{a}, \mu g / l$ & $-0.05(-0.22,0.12)$ & $0.13(-0.38,0.12)$ & $0.03(-0.02,0.08)$ & $0.03(-0.04,0.09)$ \\
\hline Lead $^{a}, \mu \mathrm{g} / \mathrm{dl}$ & $-0.04(-0.28,0.20)$ & $-0.02(-0.37 .0 .32)$ & $0.07(0.003,0.14)$ & $0.06(-0.04,0.15)$ \\
\hline \multirow[t]{3}{*}{ Mercury ${ }^{a}, \mu g / l$} & $0.002(-0.10,0.11)$ & $0.04(-0.09,0.19)$ & $-0.02(-0.05,0.004)$ & $-0.03(-0.07,0.01)$ \\
\hline & \multicolumn{4}{|l|}{ Dietary Vitamin $B_{12}$} \\
\hline & $<4.47 \mu \mathrm{g} /$ day $^{\mathrm{b}}$ & $\geq 4.47 \mu \mathrm{g} /$ day $^{\mathrm{b}}$ & $<4.47 \mu \mathrm{g} /$ day $^{\mathrm{b}}$ & $\geq 4.47 \mu \mathrm{g} /$ day $^{\mathrm{b}}$ \\
\hline Cadmium $^{\mathrm{a}}, \mu \mathrm{g} / \mathrm{I}$ & $-0.08(-0.26,0.09)$ & $-0.17(-0.40,0.05)$ & $0.02(-0.02,0.07)$ & $0.06(-0.01,0.12)$ \\
\hline Lead $^{\mathrm{a}}, \mu \mathrm{g} / \mathrm{dl}$ & $-0.02(-0.27,0.22)$ & $-0.17(-0.50,0.16)$ & $0.08(0.01,0.14)$ & $0.05(-0.03,0.14)$ \\
\hline \multirow[t]{3}{*}{ Mercury $^{a}, \mu g$ / } & $0.03(-0.08,0.13)$ & $-0.003(-0.15,0.15)$ & $-0.03(-0.06,-0.005)$ & $-0.01(-0.05,0.03)$ \\
\hline & \multicolumn{4}{|l|}{ Dietary Folate } \\
\hline & $<456 \mu \mathrm{g} /$ day $^{\mathrm{b}}$ & $\geq 456 \mu \mathrm{g} /$ day $^{\mathrm{b}}$ & $<456 \mu \mathrm{g} /$ day $^{\mathrm{b}}$ & $\geq 456 \mu \mathrm{g} /$ day $^{\mathrm{b}}$ \\
\hline Cadmium $^{\mathrm{a}}, \mu \mathrm{g} / \mathrm{I}$ & $-0.09(-0.27,0.09)$ & $-0.16(-0.36,0.04)$ & $0.02(-0.03,0.07)$ & $0.03(-0.03,0.10)$ \\
\hline Lead $^{a}, \mu \mathrm{g} / \mathrm{dl}$ & $-0.01(-0.27,0.24)$ & $0.06(-0.24,0.36)$ & $0.07(0.0004,0.14)$ & $0.06(-0.03,0.15)$ \\
\hline \multirow[t]{3}{*}{ Mercury $^{\mathrm{a}}, \mu \mathrm{g} / \mathrm{I}$} & $-0.001(-0.11,0.11)$ & $0.07(-0.05,0.20)$ & $-0.03(-0.06,0.0001)$ & $-0.03(-0.07,0.01)$ \\
\hline & \multicolumn{4}{|l|}{ Serum Folate } \\
\hline & $<24.49 \mathrm{ng} / \mathrm{ml}^{\mathrm{b}}$ & $\geq 24.49 \mathrm{ng} / \mathrm{ml}^{\mathrm{b}}$ & $<24.49 \mathrm{ng} / \mathrm{ml}^{\mathrm{b}}$ & $\geq 24.49 \mathrm{ng} / \mathrm{ml}^{\mathrm{b}}$ \\
\hline Cadmium $^{a}, \mu g / l$ & $-0.05(-0.23,0.13)$ & $-0.24(-.52,0.04)$ & $0.03(-0.02,0.08)$ & $0.09(0.02,0.15)$ \\
\hline Lead $^{\mathrm{a}}, \mu \mathrm{g} / \mathrm{dl}$ & $-0.07(-0.32,0.18)$ & $0.01(-0.39,0.42)$ & $0.07(0.0001,0.14)$ & $-0.002(-0.10,0.10)$ \\
\hline Mercury $^{a}, \mu g$ /l & $0.03(-0.08,0.13)$ & $0.03(-0.14,0.21)$ & $-0.03(-0.06,-0.005)$ & $-0.01(-0.05,0.03)$ \\
\hline
\end{tabular}

Abbreviations: hs-CRP high sensitivity C-reactive protein, $t$ Hcy total serum homocysteine

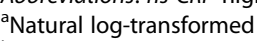

${ }^{b}$ Adjusted for age, race (black, white, other), BMI, parity, smoking, and day of menstrual cycle with inverse probability of exposure weights that included timevarying confounding: vitamins $\left(B_{6}, B_{12}\right.$, folate), reproductive hormone concentrations, calorie and alcohol intake

When simultaneously adjusting for metal coexposure, each percent increase in lead remained associated with increased homocysteine, after adjustment for relevant confounding factors $0.10 \mu \mathrm{mol} / \mathrm{L}$ (95\% CI: 0.03, 0.16) (Table 3). Cadmium and mercury were not associated with homocysteine or hs-CRP in the co-exposure models. Composite metals categories were not associated with changes in either hs-CRP or homocysteine concentrations (Table 3). A secondary analysis of mercury and homocysteine by additionally adjusting for mean fish consumption and omega-3 fatty acid levels both together and separately, which showed that the findings were robust to these additional potential confounders.

\section{Discussion}

To our knowledge, this is among the first studies to demonstrate that blood lead was associated with increases in homocysteine concentrations among healthy women with blood lead concentrations similar to or lower than the US population. In particular, the association between lead and homocysteine concentrations persisted among women consuming lower levels of essential B vitamins and folate. Specifically, geometric mean blood cadmium $(0.29 \mu \mathrm{g} / \mathrm{l}[26]$ vs. $0.29 \mu \mathrm{g} / \mathrm{l}$ in the present study), lead $(1.78 \mu \mathrm{g} / \mathrm{dl}$ [27] vs. $0.91 \mu \mathrm{g} / \mathrm{l})$, and mercury $(1.02 \mu \mathrm{g} / \mathrm{l}[28]$ vs. $1.04 \mu \mathrm{g} / \mathrm{l})$ concentrations are similar to or lower than those observed among reproductive-aged women in the US. These findings suggest that even among young, healthy women, with relatively low exposure, higher lead concentrations are associated with higher homocysteine concentrations. This association remained after adjustment using weighted models to account for time varying confounding by micronutrient intake and reproductive hormone concentrations. Importantly, micronutrient intake modified the association and our findings suggest that lead was not associated with 
Table 3 Metal co-exposure in relation to high sensitivity-C reactive protein and total plasma homocysteine

\begin{tabular}{|c|c|c|c|c|}
\hline & \multicolumn{2}{|l|}{ hs-CRP ${ }^{b}$ (mg/L) } & \multicolumn{2}{|l|}{$\mathrm{tHcy}^{\mathrm{b}}(\mu \mathrm{mol} / \mathrm{L})$} \\
\hline & Unadjusted & Adjusted $^{a}$ & Unadjusted & Adjusted $^{a}$ \\
\hline Cadmium ${ }^{\mathrm{b}}, \mu \mathrm{g} / \mathrm{I}$ & $0.18(-0.16,0.51)$ & $0.17(-0.20,0.55)$ & $0.03(-0.02,0.07)$ & $0.02(-0.03,0.07)$ \\
\hline Lead $^{b}, \mu g / d l$ & $0.25(-0.23,0.73)$ & $0.16(-0.34,0.66)$ & $0.09(0.03,0.15)$ & $0.10(0.03,0.16)$ \\
\hline Mercury ${ }^{b}, \mu g / l$ & $0.08(-0.13,0.30)$ & $0.09(-0.13,0.31)$ & $-0.02(-0.05,0.01)$ & $-0.02(-0.05,0.01)$ \\
\hline \multicolumn{5}{|l|}{ Composite $^{c}$} \\
\hline Low & Ref & Ref & Ref & Ref \\
\hline Medium & $0.26(-0.12,0.63)$ & $0.37(-0.01,0.74)$ & $0.02(-0.04,0.08)$ & $0.01(-0.04,0.07)$ \\
\hline High & $0.18(-0.23,0.59)$ & $0.19(-0.21,0.60)$ & $0.05(-0.01,0.12)$ & $0.05(-0.02,0.11)$ \\
\hline
\end{tabular}

Abbreviations: $h s-C R P$ high sensitivity C-reactive protein, $t$ Hcy total serum homocysteine

${ }^{a}$ Adjusted for age, BMI, smoking, race, average calories, cycle day and parity

${ }^{\mathrm{b}}$ Natural log-transformed

'Composite tertiles definitions: high (in highest tertile of at least two metals), low (in lowest tertile of at least two metals), medium (all others)

increased homocysteine concentrations for those with consumption of micronutrients $\geq 75$ th percentile.

Our finding that lead was associated with homocysteine is supported by several studies $[1,4,6,10,29]$, which generally had higher lead exposures (average lead range: 3.5 to $22.7 \mu \mathrm{g} / \mathrm{dL}$ vs. $0.91 \mu \mathrm{g} / \mathrm{dL}$ in the present study) and older populations (mean age: $38-59$ years vs. 27 in the present study) than the present study. A cross-sectional study among adults in Pakistan with average blood lead concentrations $11.7 \mu \mathrm{g} / \mathrm{dL}$ found greater changes in homocysteine $4.6 \%$ (95\% CI: 2.6, 4.8\%) per IQR increase $(\sim 8 \mu \mathrm{g} / \mathrm{dL})$ in lead concentrations [6]. In contrast, lead exposures in our study were about $10 \mu \mathrm{g} / \mathrm{dL}$ lower. It is therefore notable that we observed similar findings in a healthy, younger population. A cross-sectional study of US adults, who were older than in the present study (mean age 50 vs. 27 years), found that the association between lead and homocysteine was modified by essential micronutrient concentrations [5]. Like Lee et al., who observed stronger associations between lead and homocysteine among those with lower concentrations of vitamin $B_{6}$ and folate, here, the positive association between lead and homocysteine persisted among those with vitamin $B_{6}, B_{12}$, dietary and serum folate concentrations below the 75th percentile [5]. This is notable given differences by population age composition, sex, or concentration of homocysteine, as no one in the present study had concentrations that would be categorized as high $(>15 \mu \mathrm{mol} / \mathrm{L})$. A longitudinal study of older men (mean 69 years) with higher blood lead concentrations ( 4.9 vs. $0.91 \mu \mathrm{g} / \mathrm{dl})$ similarly found that lead and homocysteine were more strongly associated among those with lower concentrations of folate and B vitamins [10], which was consistent with our findings of those below the 75th percentile of folate and B vitamins. Thus, our findings support the hypothesis that higher consumption of essential micronutrients may mitigate the observed association between lead and homocysteine among reproductive-aged women with low lead concentrations. This is striking given the health status of study participants and the relatively low concentrations of lead and homocysteine, along with fairly moderate levels of micronutrient intake. In particular, participants were selected who did not report special diets, which may explain the lower vitamin intake.

Mechanistically, lead may affect homocysteine concentrations via several pathways. Lead has been associated with oxidative stress, irregularities in the nitric oxide system, and inflammation [30,31]. Lead can also interact with sulfhydryl groups, with homocysteine being a sulfur-containing amino acid [1, 32]. Lead exposure could lead to copper deficiency [33], and diets high in copper were associated with slight declines in folate and homocysteine concentrations [34], which may imply that diets low in copper could also affect homocysteine concentrations.

Mercury was associated with decreased homocysteine concentrations among those with serum folate $<24.49 \mathrm{ng} / \mathrm{ml}$. One study in children found a similar association among boys but not girls [3]. The mechanism of action by which mercury is associated with decreased concentrations of homocysteine in the presence of lower concentrations of serum folate may be by an increased need for glutathione, which causes homocysteine to be involved in glutathione production instead of methylating methionine [35]. This process would lead to lower homocysteine concentrations. Mercury has an affinity to selenium, an essential nutrient, which may play a role in its slightly negative association with homocysteine. Experimental evidence showed that plasma homocysteine was significantly reduced in selenium-deprived animals [36]. Additionally, diets rich in fish have been associated with reduced risk of coronary heart disease and fish consumption is a significant source of mercury exposure [37]. It is possible that the lower concentrations of homocysteine observed here were driven by fish intake and other lifestyle traits. While total fish 
consumption measured by 24-h dietary recall was low, median $=0$ (range 0-12.2 servings), mean fish consumption was determined for each participant considering the up to eight 24-h dietary recalls completed in the study. Mean fish consumption was positively correlated with mercury concentrations (Spearman $\rho=0.42, p<0.001$ ) and mercury was weakly associated with omega-3 fatty acid levels (Spearman $\rho=0.06 p<0.001$ ). There are several possible reasons why metals were not associated with hs-CRP. A cross-sectional population-based study investigated these associations in an older population (age range 40-79 years) and found that urinary cadmium was associated with CRP [8]. Another cross-sectional study in an older population (mean female age 43.3 years) found urinary cadmium was significantly associated with increases in CRP, which were mitigated by adjustment for a composite antioxidant dietary score [9]. However, in the present study, the population was much younger, with a mean age of 27.4 years, and we did observe associations between age and hs-CRP. In this study, $95 \%$ of participants had concentrations of cadmium below $0.86 \mu \mathrm{g} / \mathrm{l}$ and median concentrations were $0.30 \mu \mathrm{g} / \mathrm{l}$. Therefore, exposure concentrations may have been too low to observe such associations. The lack of association between CRP and metals may have been because the majority of the study population (61\%) was of normal BMI, and CRP concentrations are tied to obesity-linked chronic inflammation [38]. BioCycle participants were selected to be free of chronic disease and medication use, and this selection strategy may have contributed to the null findings with respect to CRP.

This study had several limitations. Study generalizability may be limited to healthy, reproductive-aged women with fairly low concentrations of metal exposure. Further, this study had very low smoking prevalence, and thus findings may not be generalizable to populations with higher levels of smoking. The BMI of participants in the present study was lower than average, as most participants were in the normal range, which may limit generalizability. Other vitamins and trace elements such as zinc and copper are associated with inflammatory markers and could interact with metals but unfortunately were not measured in this study. Measurement error in metals, homocysteine, hs-CRP, or micronutrients may have biased our ability to observe associations. However, the fasting early-morning measurement of homocysteine may have mitigated some of the previously reported diurnal variability [39]. Should correlated measurement error have affected our findings, it is likely that this would have resulted in bias toward the null, which may mean that true associations were stronger than observed here [40].

However, this study had several strengths. Participants in the study were selected to be free from underlying chronic conditions, medication use, or special diets [17] to minimize unmeasured confounding. Multiple measurements of homocysteine and hs-CRP is another strength, as it has been shown that hs-CRP concentrations can change even over the menstrual cycle [41]. Further, consideration of modification by dietary and serum micronutrient levels among a population that did not consume special diets, medications, or supplements, is an additional strength. The consideration of timevarying confounding and the use of marginal structural models to appropriately account for such confounding minimizes the chance that observed associations were the result of confounding bias.

\section{Conclusions}

We observed that lead was associated with increased homocysteine among healthy, young women. These findings suggest that even at low concentrations of lead exposure, among healthy women, lead may play a role in altering homocysteine concentrations, with potential implications for chronic diseases later in life. Among women with consumption of vitamins $B_{12}, B_{6}$, dietary and serum folate below the 75th percentile, lead was associated with higher concentrations of the inflammatory biomarker, homocysteine. Our findings showed that higher micronutrient concentrations may offer protection from the influence of lead on an important biomarker of cardiovascular risk.

\section{Abbreviations}

BMI: Body mass index; Cl: Confidence interval; CRP: C-reactive protein; CVD: Cardiovascular disease; hs-CRP: High-sensitivity-C-reactive protein; tHcy: Total plasma homocysteine

\section{Acknowledgements}

The authors thank the investigators and staff at the Epidemiology Branch, Eunice Kennedy Shriver National Institute of Child Health and Human

Development, and at the University at Buffalo for their respective roles in the study and the study participants for their commitment to the study.

\section{Funding}

This work was supported by the Intramural Research Program of the Eunice Kennedy Shriver National Institute of Child Health and Human Development, National Institutes of Health (Contract \# HHSN275200403394C).

\section{Availability of data and materials \\ The datasets analyzed during the current study are available from the corresponding author on reasonable request.}

\begin{abstract}
Authors' contributions
AZP conceptualized the design of the study, carried out the statistical analyses, and revised the initial manuscript. SLM, LS, NJP critically reviewed and revised the manuscript. FM carried out portions of the statistical analyses and drafted the initial manuscript. JWW was the site principal investigator of the study, and critically reviewed the manuscript. EFS conceptualized the study, was the study $\mathrm{PI}$, and critically reviewed and revised the manuscript. All authors read and approved the final manuscript.
\end{abstract}

Ethics approval and consent to participate

The University at Buffalo Health Sciences Institutional Review Board (IRB) approved the study and served as the IRB designated by the $\mathrm{NIH}$ for this 
study under a reliance agreement. All participants provided written informed consent.

\section{Consent for publication}

Not applicable.

\section{Competing interests}

The authors declare that they have no competing interests.

\section{Publisher's Note}

Springer Nature remains neutral with regard to jurisdictional claims in published maps and institutional affiliations.

\section{Author details}

${ }^{1}$ Department of Global and Community Health, College of Health and Human Services, George Mason University, 4400 University Drive MS5B7, Fairfax, VA 22030, USA. Epidemiology Branch, Division of Intramural Population Health Research, Eunice Kennedy Shriver National Institute of Child Health and Human Development, National Institutes of Health, Bethesda, MD, USA. ${ }^{3}$ Department of Epidemiology and Environmental Health, School of Public Health and Health Professions, University at Buffalo, Buffalo, NY, USA

Received: 19 January 2017 Accepted: 31 July 2017

Published online: 08 August 2017

\section{References}

1. Schafer JH, Glass TA, Bressler J, Todd AC, Schwartz BS. Blood lead is a predictor of homocysteine levels in a population-based study of older adults. Environ Health Perspect. 2005;113:31-5.

2. Guallar E, Silbergeld EK, Navas-Acien A, Malhotra S, Astor BC, Sharrett AR, et al. Confounding of the relation between homocysteine and peripheral arterial disease by lead, cadmium, and renal function. Am J Epidemiol. 2006;163:700-8

3. Gallagher CM, Meliker JR. Total blood mercury, plasma homocysteine, methylmalonic acid and folate in US children aged $3-5$ years, NHANES 1999-2004. Sci Total Environ. 2011:409:1399-405.

4. Chia SE, Ali SM, Lee BL, Lim GH, Jin S, Dong NV, et al. Association of blood lead and homocysteine levels among lead exposed subjects in Vietnam and Singapore. Occup Environ Med. 2007;64:688-93.

5. Lee Y-M, Lee M-K, Bae S-G, Lee S-H, Kim S-Y, Lee D-H. Association of homocysteine levels with blood lead levels and micronutrients in the US general population. J Prev Med Public Health Yebang Ǔihakhoe Chi. 2012;45:387-93.

6. Yakub M, lqbal MP. Association of blood lead (Pb) and plasma homocysteine: a cross sectional survey in Karachi, Pakistan. Plos One. 2010;5:e11706

7. Barregard L, Sallsten G, Fagerberg B, Borné Y, Persson M, Hedblad B, et al. Blood Cadmium Levels and Incident Cardiovascular Events during Followup in a Population-Based Cohort of Swedish Adults: The Malmö Diet and Cancer Study. Environ Health Perspect. 2016;124(5):594-600.

8. Lin Y-S, Rathod D, Ho W-C, Caffrey JJ. Cadmium exposure is associated with elevated blood C-reactive protein and fibrinogen in the U. S. population: the third national health and nutrition examination survey (NHANES III, 1988-1994). Ann Epidemiol. 2009:19:592-6.

9. Colacino JA, Arthur AE, Ferguson KK, Rozek LS. Dietary antioxidant and antiinflammatory intake modifies the effect of cadmium exposure on markers of systemic inflammation and oxidative stress. Environ Res. 2014;131:6-12.

10. Bakulski KM, Park SK, Weisskopf MG, Tucker KL, Sparrow D, Spiro A, et al. Lead exposure, B vitamins, and plasma homocysteine in men 55 years of age and older: the VA normative aging study. Environ Health Perspect. 2014:122:1066-74

11. Strain JJ, Dowey L, Ward M, Pentieva K, McNulty H. B-vitamins, homocysteine metabolism and CVD. Proc Nutr Soc. 2004;63:597-603.

12. Eaker ED, Chesebro JH, Sacks FM, Wenger NK, Whisnant JP, Winston M. Cardiovascular disease in women. Circulation. 1993:88:1999-2009.

13. Go AS, Mozaffarian D, Roger VL, Benjamin EJ, Berry JD, Blaha MJ, et al. Heart disease and stroke statistics-2014 update: a report from the American Heart Association. Circulation. 2014;129:e28-292.

14. Homocysteine Studies Collaboration. Homocysteine and risk of ischemic heart disease and stroke: a meta-analysis. JAMA. 2002;288:2015-22.
15. Refsum H, Ueland PM, Nygård O, Vollset SE. Homocysteine and cardiovascular disease. Annu Rev Med. 1998:49:31-62.

16. Ridker PM, Hennekens CH, Buring JE, Rifai N. C-reactive protein and other markers of inflammation in the prediction of cardiovascular disease in women. N Engl J Med. 2000;342:836-43.

17. Wactawski-Wende J, Schisterman EF, Hovey KM, Howards PP, Browne RW, Hediger $\mathrm{M}$, et al. BioCycle study: design of the longitudinal study of the oxidative stress and hormone variation during the menstrual cycle. Paediatr Perinat Epidemiol. 2009;23:171-84.

18. Rifai N, Ballantyne CM, Cushman M, Levy D, Myers GL. Point: high-sensitivity C-reactive protein and cardiac C-reactive protein assays: is there a need to differentiate? Clin Chem. 2006:52:1254-6.

19. Pearson TA, Mensah GA, Alexander RW, Anderson JL, Cannon RO, Criqui M, et al. Markers of inflammation and cardiovascular disease: application to clinical and public health practice: a statement for healthcare professionals from the Centers for Disease Control and Prevention and the American Heart Association. Circulation. 2003;107:499-511.

20. Ridker PM. Clinical application of C-reactive protein for cardiovascular disease detection and prevention. Circulation. 2003;107:363-9.

21. Richardson DB, Ciampi A. Effects of exposure measurement error when an exposure variable is constrained by a lower limit. Am J Epidemiol. 2003:157:355-63.

22. Schisterman EF, Vexler A, Whitcomb BW, Liu A. The limitations due to exposure detection limits for regression models. Am J Epidemiol. 2006;163:374-83.

23. Cole SR, Hernán MA. Constructing inverse probability weights for marginal structural models. Am J Epidemiol. 2008;168:656-64.

24. Robins JM, Hernán MA, Brumback B. Marginal structural models and causal inference in epidemiology. Epidemiology. 2000;11:550-60.

25. Myers GL, Rifai N, Tracy RP, Roberts WL, Alexander RW, Biasucci LM, et al. CDC/AHA workshop on markers of inflammation and cardiovascular disease application to clinical and public health practice: report from the laboratory science discussion group. Circulation. 2004;110:e545-9.

26. Mijal RS, Holzman CB. Blood cadmium levels in women of childbearing age vary by race/ethnicity. Environ Res. 2010;110:505-12.

27. Lee M-G, Chun OK, Song WO. Determinants of the blood lead level of US women of reproductive age. J Am Coll Nutr. 2005;24:1-9.

28. Schober SE, Sinks TH, Jones RL, Bolger PM, McDowell M, Osterloh J, et al. Blood mercury levels in US children and women of childbearing age, 19992000. JAMA. 2003:289:1667-74

29. Kasperczyk S, Błaszczyk I, Dobrakowski M, Romuk E, Kapka-Skrzypczak L, Adamek M, et al. Exposure to lead affects male biothiols metabolism. Ann Agric Environ Med. 2013;20:721-5.

30. Stohs SJ, Bagchi D. Oxidative mechanisms in the toxicity of metal ions. Free Radic Biol Med. 1995;18:321-36.

31. Vaziri ND. Mechanisms of lead-induced hypertension and cardiovascular disease. Am J Physiol Heart Circ Physiol. 2008;295:H454-65.

32. Perna AF, Ingrosso D, Lombardi C, Acanfora F, Satta E, Cesare CM, et al. Possible mechanisms of homocysteine toxicity. Kidney Int. Suppl. 2003; 63(84):S137-140

33. Klauder DS, Petering HG. Anemia of lead intoxication: a role for copper. J Nutr. 1977;107:1779-85.

34. Tamura T, Turnlund JR. Effect of long-term, high-copper intake on the concentrations of plasma homocysteine and B vitamins in young men. Nutr Burbank Los Angel Cty Calif. 2004:20:757-9.

35. Lee D-H, Jacobs DR, Porta M. Hypothesis: a unifying mechanism for nutrition and chemicals as lifelong modulators of DNA hypomethylation. Environ Health Perspect. 2009;117:1799-802.

36. Uthus EO, Ross S. Dietary selenium (se) and copper (cu) interact to affect homocysteine metabolism in rats. Biol Trace Elem Res. 2009;129:213-20.

37. Guallar E, Sanz-Gallardo MI, van't Veer P, Bode P, Aro A, Gomez-Aracena J, et al. Mercury, fish oils, and the risk of myocardial infarction. N Engl J Med. 2002:347:1747-54

38. Visser M, Bouter LM, McQuillan GM, Wener MH, Harris TB. Elevated C-reactive protein levels in overweight and obese adults. JAMA. 1999;282:2131-5.

39. Bönsch D, Hothorn T, Krieglstein C, Koch M, Nehmer C, Lenz B, et al. Daily variations of homocysteine concentration may influence methylation of DNA in normal healthy individuals. Chronobiol Int. 2007;24:315-26. 
40. Pollack AZ, Perkins NJ, Mumford SL, Ye A, Schisterman EF. Correlated biomarker measurement error: an important threat to inference in environmental epidemiology. Am J Epidemiol. 2013;177:84-92.

41. Gaskins AJ, Wilchesky M, Mumford SL, Whitcomb BW, Browne RW,

Wactawski-Wende J, et al. Endogenous reproductive hormones and C-reactive protein across the menstrual cycle: the BioCycle study. Am J Epidemiol. 2012;175:423-31.

Submit your next manuscript to BioMed Central and we will help you at every step:

- We accept pre-submission inquiries

- Our selector tool helps you to find the most relevant journal

- We provide round the clock customer support

- Convenient online submission

- Thorough peer review

- Inclusion in PubMed and all major indexing services

- Maximum visibility for your research

Submit your manuscript at www.biomedcentral.com/submit 